PSICOLOGÍA

IBEROAMERICANA
Psicología Iberoamericana ISSN: 1405-0943

revista.psicologia@ibero.mx

Universidad Iberoamericana, Ciudad de México México

\title{
Acción colectiva en contra de la violencia hacia las mujeres, un análisis de redes bayesianas
}

Zamudio Sosa, Alejandro; Montero-Lopéz Lena, María

Acción colectiva en contra de la violencia hacia las mujeres, un análisis de redes bayesianas

Psicología Iberoamericana, vol. 29, núm. 2, e292350, 2021

Universidad Iberoamericana, Ciudad de México, México

Disponible en: https://www.redalyc.org/articulo.oa?id=133967822002

DOI: https://doi.org/10.48102/pi.v29i2.350

Los autores/as conservan los derechos de autor y ceden a la revista el derecho de la primera publicación, con la obra registrada bajo la Licencia Creative Commons Atribución 4.0 Internacional.

Los autores/as conservan los derechos de autor y ceden a la revista el derecho de la primera publicación, con la obra registrada bajo la Licencia Creative Commons Atribución 4.0 Internacional.

\section{(c) (i)}

Esta obra está bajo una Licencia Creative Commons Atribución 4.0 Internacional. 


\title{
Artículos
}

\section{Acción colectiva en contra de la violencia hacia las mujeres, un análisis de redes bayesianas}

Collective action against violence against women, an analysis of Bayesian networks

\author{
Alejandro Zamudio Sosa zamudiososaalejandro@gmail.com \\ Universidad Nacional Autónoma de México (UNAM), México \\ (D) https://orcid.org/0000-0002-3902-5585 \\ María Montero-Lopéz Lena monterol@unam.mx \\ Universidad Nacional Autónoma de México (UNAM), México \\ https://orcid.org/0000-0001-9109-381X
}

Psicología Iberoamericana, vol. 29, núm. 2, e292350, 2021

Universidad Iberoamericana, Ciudad de México, México

Recepción: 22 Febrero 2021 Aprobación: 04 Agosto 2021

DOI: https://doi.org/10.48102/ pi.v29i2.350

Redalyc: https://www.redalyc.org/ articulo.oa?id $=133967822002$

Financiamiento

Fuente: Consejo Nacional de Ciencia y

Tecnología (CONACYT)

No de contrato: 925505

Beneficiario: Alejandro Zamudio Sosa
Resumen: En el estudio de la acción colectiva existen múltiples modelos explicativos, sin embargo, no se conocen un modelo en el contexto de la acción colectiva en contra de la violencia hacia las mujeres en México. Por ello, el propósito del presente estudio fue explorar cómo se relacionan la identidad feminista, de género, autoeficacia, expectativas de resultados, eficacia colectiva, percepción de injusticia, indignación, norma subjetiva y las acciones colectivas a partir de una muestra de 488 mujeres de la Zona Metropolitana de la Ciudad de México obtenida en octubre del 2019. Con un muestro no probabilístico, mediante redes sociales, se aplicaron instrumentos creados para la presente investigación. Con análisis de redes latentes y aprendizaje de redes bayesianas, se encontró que la norma subjetiva y la indignación tomaron papeles de centralidad entre las variables estudiadas, mientras que la percepción de injusticia tuvo influencia indirecta sobre la acción. La norma subjetiva y la percepción de injusticia pudieran ser clave para entender la acción colectiva en el contexto de violencia hacia las mujeres en México.

Palabras clave: acción colectiva, identidad colectiva, percepción de injusticia, norma subjetiva, mujeres.

Abstract: In the study of collective action, there are multiple explanatory models, however, there is no known model in the context of collective action against violence against women in Mexico. Therefore, the purpose of this study was to explore how feminist identity, gender, self-efficacy, expectations of results, collective efficacy, perception of injustice, indignation, subjective norms, and collective actions are related amongst 488 women from the Metropolitan Area of Mexico City, obtained in October 2020. Participants were drawn from a non-probabilistic sample via social networks and instruments created for the present investigation tested on participants. Through analysing latent networks and learning of Bayesian networks, it was found that the subjective norms and indignation took central roles among the variables studied, while the perception of injustice had an indirect influence on the action. The subjective norms and the perception of injustice could be key to understanding collective action in the context of violence against women in Mexico.

Keywords: collective action, collective identity, perception of injustice, subjective norm, women. 


\section{Introducción}

La violencia hacia las mujeres es uno de los temas que más ha tenido atención de la opinión pública en México en las últimas décadas. Se estima que en México entre 9 y 10 mujeres son asesinadas todos los días, ya para el 2015 se había emitido alerta de género por el número de asesinados en el Estado de México (Madrid, 2019) y en el 2019 ocurrió la misma situación en la Ciudad de México (Gobierno de la Ciudad de México, 2019), siendo así el feminicidio la expresión más grave de la violencia hacia las mujeres en México (Lagarde, 2011). Aunado a esto, Romero Mendoza et al. (2021) han reportado que la violencia hacia las mujeres en México tiene un impacto social y también general en la salud de las mujeres que la sufren. Pedraza (2019) considera que la violencia hacia las mujeres en México es tan generalizarla que debe ser considerado una emergencia nacional, y por en ende se deben implementar acciones desde diferentes frentes para buscar erradicarla. Ante esta situación, en los últimos años han aumentado las acciones colectivas e individuales que las mismas mujeres han realizado con el fin de visualizar, tipificar e influir en la política y en la sociedad para disminuir dicha violencia. De acuerdo con el Laboratorio de Análisis de Organizaciones y Movimientos Sociales de la UNAM (CEIICH-UNAM, 2020), desde el 2015 las protestas de las mujeres en México han aumentado anualmente, siendo el año 2019 el de mayores protestas por parte de las mujeres con un total de 66. En este sentido, Hincapié (2017) ha reportado que el tema de los feminicidios en México ha provocado procesos crecientes de acción colectiva por parte de las mujeres, fortaleciendo identidades de género y feministas, marcos de injusticia basados en derechos humanos, y dirigiendo sus objetivos al cumplimiento de acciones efectivas por parte de las autoridades estatales o federales para la resolución y justicia de dichos casos. Consignas como ni una menos (Ramírez, 2018; Sosa, 2016) es ejemplo de cómo los feminicidios han sido uno de los principales motivadores de la creciente acción colectiva por parte de las mujeres. Dunckel Graglia $(2013,2016)$ ha reportado que la acción colectiva de mujeres en México puede ayudar enmarcar problemas habituales como el acoso en el trasporte público a pasar a ser visto como un "problema de género" y de violencia hacia las mujeres. Chen (2014) considera que en la Ciudad de México la institucionalización de movimientos de mujeres (institutos gubernamentales y no gubernamentales) lejos de llevar a las nuevas generaciones a un estado de complacencia, sienta las bases para sensibilización y fortalecimiento de identidades colectivas en mujeres jóvenes y la consolidación de una conciencia feminista. Por su parte, Cerva Cerna (2020) considera que la violencia hacia las mujeres ha sido el principal tema del surgimiento de nuevos actores políticos y de nuevos colectivos que surgen en las universidades mexicanas que tienen impacto más allá de las mismas, y cuya identidad colectiva y repertorios de acción resultan cualitativamente diferentes a otros colectivos. De este modo, en la acción colectiva de las mujeres, las identidades de género y feministas parecen resultar relevantes (Cerva Cerna, 2020; Ramírez, 2018; Sancho, 
2018), pero no solo eso, otros factores como la conceptualización de la injusticia o diferentes tipos de emociones pueden jugar también un papel importante (Hincapié, 2017).

En psicología social, existe una amplia tradición en el estudio de la participación en acciones colectivas y uno de los primeros y principales constructos con los cuales se ha asociado es la identidad colectiva (Polletta \& Jasper, 2001). En el contexto de acciones colectivas de mujeres, investigaciones han encontrado que las mujeres con alta identificación feminista (Chen, 2014; Liss et al., 2004; Nelson et al., 2008; Sancho, 2018; Zucker, 2004) o hacia el sexo (Kelly \& Breinlinger, 1995) tienen más probabilidad de participar en acciones colectivas.

Otro de los constructos altamente estudiados en este contexto es el de percepción de injusticia (Clay-Warner, 2001), el cual ha sido relacionado con la identidad colectiva en varios estudios (Kelly \& Breinlinger, 1995, 1996). En sociología se ha tomado en cuenta un constructo similar, a saber, los marcos de interpretación. Estos marcos permiten dar sentido al mundo, siendo el marco de diagnóstico aquel que permite a las personas a reconocer hechos como problemas sociales, sus orígenes y sus consecuencias (Delgado \& Arias, 2018; Simon \& Klandermans, 2001). Aunado a estos marcos, y al ser cada vez más evidente la influencia de las emociones en la acción colectiva (Poma \& Gravante, 2013), estas se fueron incorporando en el estudio de la acción colectiva, y principalmente de las llamadas emociones basadas en grupo (Mackie et al., 2008) las cuales han sido incluidas en teorías y modelos explicativos de la acción colectiva (Rees \& Bamberg, 2014; Sabucedo et al., 2010). Así, la indignación y el enojo han sido las emociones que más se han incluido en este tipo de modelos (Leach et al., 2006; Stümer \& Simon, 2009). Dichas emociones son frecuentemente asociadas a la percepción de injusticia o a los marcos de injusticia.

La relación entre la injusticia y la identidad colectiva puede variar en función de la forma en que se enmarcan en un primer lugar una problemática. En este sentido, Tajfel (1978) sostiene que las personas que cuentan con una identidad de grupo determinada y que se encuentra en una situación de desventaja pueden emprender tres principales estrategias para hacer frente a dicha situación. La primera de ellas es la movilidad social, es decir, el miembro desfavorecido puede alejarse o disociarse del grupo con el fin de deshacerse del estigma considerándose diferente al grupo. La auto estereotipación, donde el miembro desfavorecido acentúa los valores positivos del grupo, y al mismo tiempo resalta los valores negativos de los grupos externos. Por último, la competencia social, donde el miembro busca elevar el estatus del grupo.

En una línea similar, Breinlinger y Kelly (1994) encontraron en mujeres dos patrones generales en las respuestas ante la desigualdad percibida. El primero, nombrado movilidad individual, que se caracteriza por ideas de que el éxito puede estar relacionado con las características individuales y donde la identificación con otras mujeres se minimiza y se disocian psicológicamente con el estado de desigualdad de las mujeres. El segundo, llamado cambio social, donde existe alta identidad social y se 
cree que las mujeres deben de actuar en grupo para promover los cambios sociales. Aquí, las mujeres expresan un fuerte sentido de solidaridad con otras mujeres y ven a la acción colectiva como la única manera de lograr igualdad para las mujeres.

Por otra parte, las creencias de eficacia y eficacia colectiva han sido otra de las variables que más se han estudiado en el contexto de la acción colectiva (Eisele \& Stake, 2008; Van Zomeren et al., 2013, 2004). La percepción de injusticia, la eficacia colectiva y la identidad han sido objeto de estudio en un meta análisis demostrando su efecto sobre la participación en acciones colectivas (Van Zomeren et al., 2008).

En cuanto a la relación entre la percepción de injusticia y la eficacia colectiva, hay por lo menos tres hipótesis de relación. La primera sostiene que la relación es negativa, dado que las creencias de eficacia pudieran aumentar las probabilidades de pensar que un cambio se dará independientemente de la participación (Folger, 1986). La segunda sostiene que la relación es positiva, dado que las fuertes creencias de eficacia permiten a las personas experimentar injusticia (Mackie et al., 2000). La tercera sostiene que la injustica y la eficacia son relativamente independientes entre sí (Van Zomeren et al., 2004).

Por otro lado, variables de tipo normativo como la norma subjetiva (entendida como las percepción de las expectativas de personas cercanas sobre determinado comportamiento y la motivación para cumplir dichas expectativas; Ajzen, 1991) han sido poco estudiadas (Bamberg et al., 2015). Rees y Bamberg (2014) integraron el constructo de normas sociales en el modelo de identidad social de la acción colectiva mostrando fuerte influencia y adecuados índices de ajuste. Por su parte, Stürmer y Simon (2004) encontraron que una variable normativa era fuertemente predictora de la acción colectiva en el contexto del movimiento gay en Alemania.

De este modo, existen propuestas de modelos explicativos que incluyen tres o más de las variables antes mencionadas, como el desarrollado por Van Zomeren et al. (2013) llamado SIMCA (social identity model of collective action), el cual plantea que la identidad social influye de forma indirecta en la intención de participar a través de las creencias de eficacia colectiva, eficacia participativa y emociones negativas basadas en el grupo. Dicho modelo ajustó adecuadamente a los datos en una muestra de adultos en países bajos. Otro de los modelos en el estudio de la acción colectiva que incluyen este tipo de variables ha sido el planteado por Van Stekelenburg et al. (2009), los autores propusieron que la identidad social influía en las acciones de protesta a través de tres vías, mediante un factor instrumental (daño percibido y eficacia), a través de la ideología y a través del enojo basado en el grupo.

Existen varios modelos que buscan explicar la participación en acciones colectivas y que toman de diferente forma varios de los constructos antes mencionados (Groenendyk \& Banks, 2014; Leach et al., 2006; Sabucedo et al., 2018; Thomas et al., 2012; Van Stekelenburg et al., 2009; Van Zomeren et al., 2008, 2013) mostrando buenos ajustes en la mayoría de los estudios. Sin embargo, en muchos de los modelos sometidos a prueba 
las relaciones de dependencia e independencia entre las variables cambian de modelo a modelo --con la excepción de la identidad social al ser, casi uniformemente, considerada como variable independiente o exógena en dichos modelos--. Así, la mayoría de modelos de relaciones entre estas variables han sido sometidas a prueba mediante modelos de ecuaciones estructurales, las cuales tiene una lógica confirmatoria y de las que se tiene evidencia que diferentes modelos, dentro del estudio de la acción colectiva, pueden ajustarse adecuadamente aún con los mismos datos (Wlodarczyk et al., 2017). Aunado a esto, aunque existen aproximaciones conceptuales (Hincapié, 2017), no se conocen modelos explicativos de la acción colectiva en el contexto de lucha en contra de la violencia hacia las mujeres en México.

Ante esta situación, surge la pregunta: ¿cómo se relacionan las variables de percepción de injusticia, indignación, identidad de género, identidad feminista, eficacia colectiva, autoeficacia, expectativas de resultados y participación en acciones colectivas, en una muestra de mujeres de la Zona Metropolitana de la Ciudad de México (ZMCDMX)?

A partir de lo expuesto anteriormente, el presente estudio tiene por objetivo explorar las relaciones entre percepción de injusticia, indignación, identidad de género, identidad feminista, eficacia colectiva, autoeficacia, expectativas de resultados y participación en acciones colectivas en el contexto de violencia hacia las mujeres en una muestra de mujeres mayores de edad de la Zona Metropolitana de la Ciudad de México (CDMX). Para dicho objetivo se utilizaron técnicas basadas en datos, a saber, análisis de redes de correlaciones parciales (algoritmo LASSO; Friedman et al., 2014) y aprendizaje con redes bayesianas (gráfico acíclico dirigido; Scutari, 2010).

Las redes de correlaciones parciales han sido usadas para explorar la relación entre síntomas en al ámbito clínico (Borsboom \& Cramer, 2013; McNally et al., 2017). Sin embargo, dichas técnicas también pueden dar cuenta de las relaciones entre constructos, así como información sobre los índices de centralidad --grado o fuerza de centralidad, centralidad de cercanía y centralidad de intermediación--, que puede dar evidencia de cómo se están correlacionando los constructos ajustando las posibles influencias espurias. Por otra parte, técnicas de gráficos acíclicos dirigidos pueden ayudar a estimar relaciones direccionadas o dirigidas entre constructos (McNally et al., 2017).

De forma general, se espera que las variables asociadas a la eficacia, la injusticia, la indignación y la normal subjetiva medien la relación entre las identidades (feminista y de género) y la participación ciudadana. En el análisis de correlaciones parciales se espera que todas las variables incluidas en el estudio se correlacionen de forma positiva en la red. Al mismo tiempo se espera que las variables asociadas a la eficacia se correlacionen fuertemente o moderadamente entre ellas. Del mismo modo, se espera que la identidad feminista se correlacione fuerte o moderadamente con la identidad de género y por su parte la percepción de injustica con la indignación. Al mismo tiempo, por ser propuestas como variables mediadoras en varios modelos (Kelly \& Breinlinger, 1996; Rees 
\& Bamberg, 2014; Van Stekelenburg et al., 2009; Van Zomeren et al., 2013) se espera que la eficacia colectiva, la autoeficacia, las expectativas de resultado, la percepción de injusticia y la indignación muestren índices de centralidad de intermediación más altos que el resto de las variables. De la misma manera se espera que la norma subjetiva tome valores más altos de centralidad de intermediación en comparación de la identidad y la acción colectiva, al ser propuesta como variable mediadora (Rees $\&$ Bamberg, 2014). En cuanto a la red bayesiana se refiere, hay una expectativa de que la identidad feminista y de género sean consideradas por la red bayesiana como nodos padres (es decir, variables que no son influidas pero que sí influyen en otras variables). Al mismo tiempo, se espera que las identidades influyan de forma indirecta en la participación en acciones colectivas mediante las creencias relacionadas con la eficacia (eficacia colectiva, autoeficacia y expectativas de resultados), la percepción de injusticia y la indignación.

\section{Método}

\section{Participantes}

La muestra estuvo conformada 488 mujeres mayores de edad habitantes de la Zona Metropolitana de la Ciudad de México (ZMCDMX). De la muestra obtenida, un $54 \%$ contaban con estudios de licenciatura, un 29\% contaba con estudios de bachillerato, un $14 \%$ con estudios de posgrados y el resto se repartía entre primaria, secundaria y sin estudios, mientras que la media de edad fue de 27.2 años con una desviación estándar de 7.8 años, en un rango de 18 a 60 años de edad. Los criterios inclusión considerados para la presente investigación fueron ser mujer, vivir en la Zona Metropolitana de la Ciudad de México (ZMCDMX) y ser mayor de edad. Para los criterios de exclusión se considero no poder contestar la encuesta por cuenta propia y haberla contestando fuera de los 10 días de aplicación establecidos.

\section{Instrumentos}

Con el fin de contar con instrumentos psicométricos válidos y confiables que dieran cuenta de las variables referidas, se generaron ítems con escala tipo Likert para evaluar los constructos mencionados, a partir de una matriz de especificaciones creada para tal propósito. Para la identidad feminista (alpha $=.76)$, la identidad de género $($ alpha $=.71)$, la eficacia colectiva (alpha $=.83)$, la autoeficacia $($ alpha $=.64)$ y las expectativas de resultados (alpha $=.63$ ) se redactaron tres reactivos para cada constructo. Mientras que para la percepción de injusticia (alpha $=.88$ ) y la indignación (alpha $=.87)$ fueron redactados dos reactivos para cada uno dada su menor complejidad. Por último, para evaluar la participación en acciones colectivas fueron redactados siete reactivos que medían la frecuencia con que las mujeres participaron, en el último año, en protestas o 
manifestaciones de forma física o de forma virtual, así como en colectivos u organizaciones con el fin de incidir en la problemática de violencia hacia las mujeres en México.

\section{Procedimiento}

Se utilizó un diseño de investigación de corte cuantitativo y trasversal para la presente investigación. Con un muestreo no probabilístico intencional y mediante redes sociales y a través de Google Formularios se difundió un formulario con los instrumentos elaborados para medir los constructos antes mencionados en un periodo de 10 días. En dicho formulario, se presentó el propósito del estudio, así como un consentimiento de participación en el mismo, en los casos donde las participantes seleccionarán no estar de acuerdo con el consentimiento la encuesta se terminaba. En el caso contrario se les presentaban instrucciones y los reactivos redactados para la presente investigación. El estudio se sujetó al Código Helsinki y las normas éticas de la investigación de la American Psychological Association (APA). Las mujeres participaron de forma voluntaria y sin recompensa de por medio.

\section{Análisis de datos}

El primer análisis consistió en calcular una red de correlaciones parciales controlando la influencia de otras posibles relaciones entre cada par de nodo. Se implementó un modelo LASSO (Friedman et al., 2014), en el que las correlaciones parciales pequeñas se trasforman a cero y no aparecen en el gráfico, de tal forma que las correlaciones parciales finales se encuentran regularizadas. Para dicho procedimiento se utilizó el software R, el paquete qgraph (Epskamp et al., 2012) y el paquete glasso (Friedman et al., 2014). Tomando en cuenta los procedimientos usado por Beard et al. (2016) y por McNally et al. (2017), se estableció el parámetro lamba en .5; así la red estima 100 diferentes modelos. También se tomó en cuenta el índice EBIC para determinar cuál red maximiza las probabilidades de tener uniones genuinas minimizando las posibles relaciones espurias (Epskamp \& Friend, 2018). Al mismo tiempo se calculó la centralidad de fuerza, de cercanía y de intermediación estandarizadas para todas las variables, el primer índice muestra la suma de todos los bordes conectados a un nodo. El segundo indica que tan cerca está un nodo de todos los demás en función de las rutas más cortas. El tercereo indica el número de veces que un nodo se encuentra en el camino más corto entre otros dos nodos, en otras palabras, la cantidad de interrelaciones que "controla" (Mair, 2018; McNally et al., 2017).

Para la red bayesiana, completamente dirigida y acíclica, se utilizó el algoritmo Hill-climbing del paquete bnlearn (Scutari, 2010). El procedimiento del algoritmo consiste en agregar, eliminar e invertir la dirección de las uniones dirigidas (flechas en el gráfico) hasta obtener la red con mejor bondad de ajuste (utilizando la métrica BIC). De esta 
forma, se realizan iteraciones hasta calcular la estructura de la red que mejor se ajuste a los datos (10 restauraciones y 100 perturbaciones para el presente estudio) con base en las distribuciones de probabilidad. Es importante resaltar que las direcciones encontradas por la red bayesiana no deben de interpretarse como relaciones de causalidad (Mair, 2018). En vez de ello se recomienda hablar de direccionalidad, sin embargo, estas direcciones pueden dar evidencia de posibles relaciones causales entre variables (Scutari \& Denis, 2015).

\section{Resultados}

Como se muestra en la Figura 1, en la red de correlaciones parciales, las creencias relacionadas con la eficacia se correlacionaron positivamente y moderadamente entre ellas, siendo la relación entre eficacia colectiva y expectativas de resultados la más fuerte. Por su parte, y como se esperaba, la indignación y la injusticia se correlacionaron fuertemente de manera positiva. Así mismo, la participación en acciones colectivas se relacionó en orden de fuerza con la identidad feminista, la norma subjetiva, la autoeficacia y la indignación. La identidad de género se relacionó débilmente con la norma subjetiva y con la identidad feminista. También se puede observar que la autoeficacia "controló” la relación entre la participación y la eficacia colectiva y las expectativas de resultados.

\section{Figura 1}

\section{Red de correlaciones parciales entre los constructos medidos}

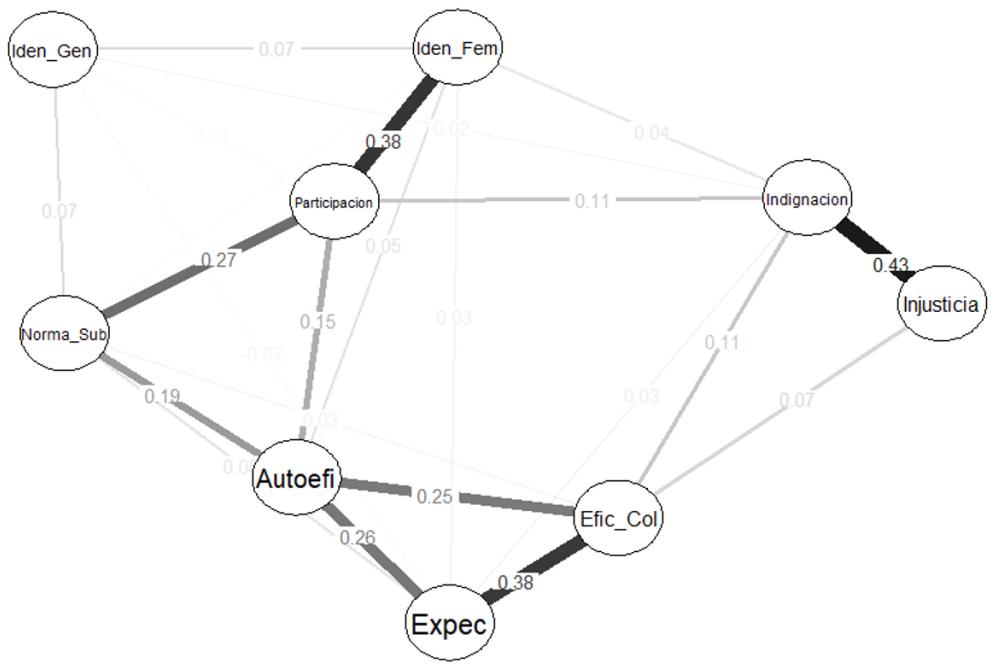

Nota. Se utilizaron las abreviaciones siguientes; a) Iden Gen para la identidad de género, b) Iden_Fem para la identidad feminista, c) Norma_Sub para la norma subjetiva, d) Autoefi para la autoeficacia, f) Efic_Col para la eficacia colectiva, g) Expec para las expectativas de resultados de las acciones colectivas.

En la Figura 2, se pueden observar los índices de centralidad de fuerza, de cercanía y de intermediación. La identidad de género es la variable con 
menos centralidad de fuerza y de cercanía. Es decir, la identidad de género fue la variable con menos relaciones y fue la que más alejada estuvo del conjunto de variables de la red. Por su parte, la participación tuvo altos niveles de centralidad de fuerza, de cercanía y de intermediación. Fue una variable con alto número de conexiones en la red, con gran cercanía a todas las demás y la variable con el mayor control de interacciones con las demás (es decir, fue la variable que más conectaba a las otras variables). También se puede observar que la autoeficacia y la indignación fueron variables con alta centralidad de intermediación, siendo congruente con la red dado que la autoeficacia controlaba la relación entre la eficacia colectiva, las expectativas de eficacia y la participación. Por su parte, la indignación controlaba la relación entre la participación y la percepción de injusticia. Por último, la identidad feminista fue una variable con moderados índices de centralidad de fuerza, de cercanía y de intermediación, siendo este último índice el más alto, lo cual significa que la identidad feminista tuvo como principal característica controlar las relaciones entre las variables de la red.

\section{Figura 2}

\section{Índices estandarizados de fuerza de centralidad, de cercanía e intermediación para} los constructos medidos

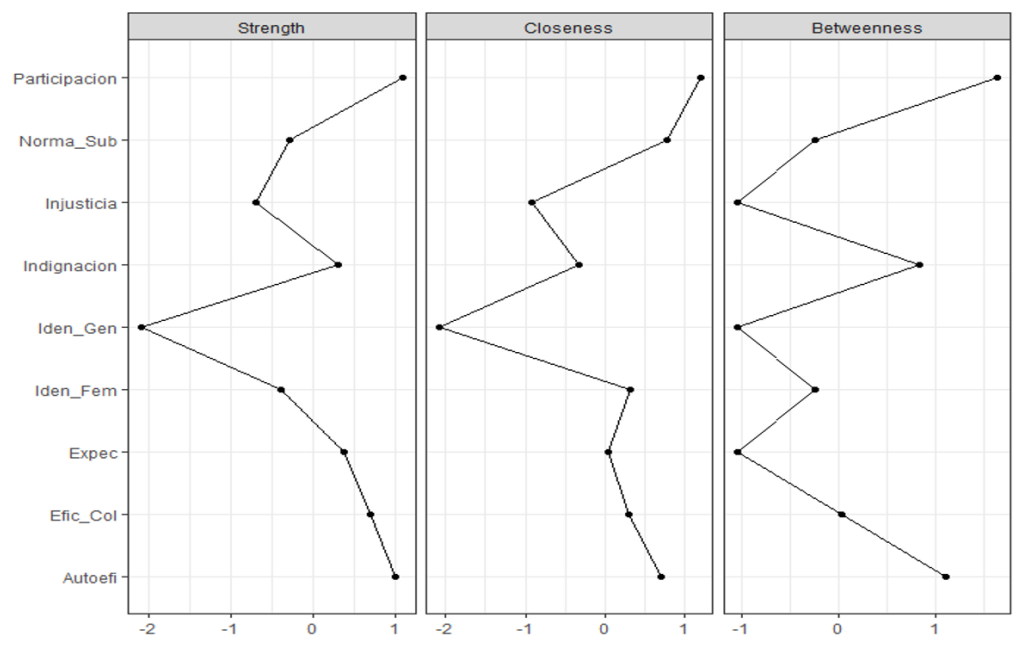

Nota. Se utilizaron las abreviaciones siguientes; a) Iden_Gen para la identidad de género, b) Iden_Fem para la identidad feminista, c) Norma_Sub para la norma subjetiva, d) Autoefi para la autoeficacia, f) Efic_Col para la eficacia colectiva, g) Expec para las expectativas de resultados de las acciones colectivas.

En el gráfico de la red acíclica (red bayesiana; Figura 3) se muestran las relaciones de dependencia probabilística, mientras más gruesa es la flecha entre dos nodos, más importante es la conexión para el buen ajuste de la red. En la Figura 3 se puede observar que la percepción de injusticia se desprenden una conexión débil hacia la eficacia colectiva y una fuerte hacia la indignación. De la eficacia colectiva se desprenden una flecha fuerte hacia la autoeficacia, una con fuerza media hacia las expectativas de 
resultados y una débil hacia la indignación. Al mismo tiempo, se puede observar que la participación en acciones colectivas es influida por la autoeficacia y la norma subjetiva directamente y por la injusticia y la eficacia colectiva indirectamente (a través de la autoeficacia). Por último, se puede observar que la participación en acciones colectivas influyó sobre la indignación de forma débil y sobre la identidad feminista de forma fuerte para la red.

\section{Figura 3}

\section{Gráfico de red bayesiana para los constructos medidos}

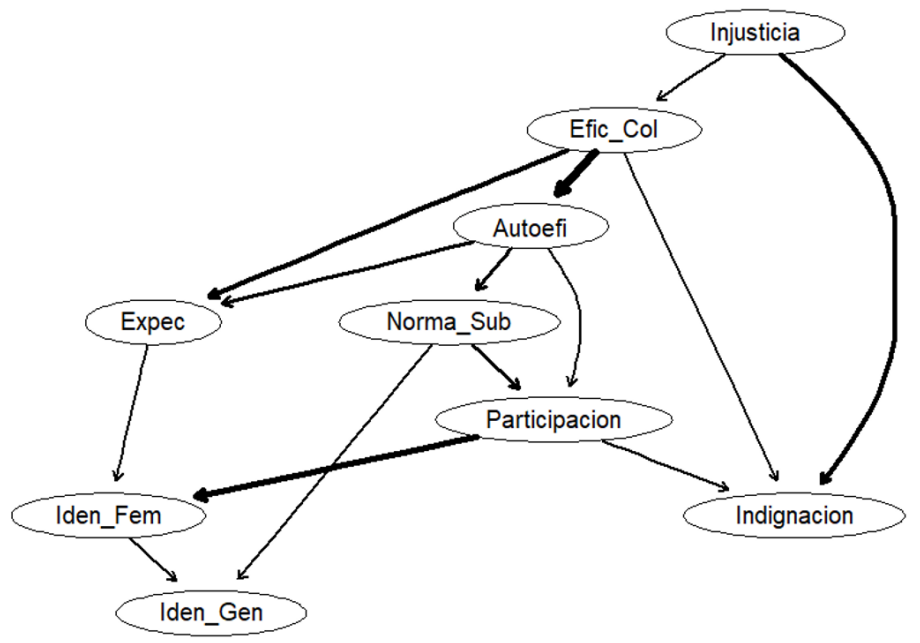

Nota. Se utilizaron las abreviaciones siguientes; a) Iden_Gen para la identidad de género, b) Iden_Fem para la identidad feminista, c) Norma_Sub para la norma subjetiva, d) Autoefi para la autoeficacia, f) Efic_Col para la eficacia colectiva, g) Expec para las expectativas de resultados de las acciones colectivas.

En la Tabla 1 se muestran los coeficientes de la fuerza de las flechas en orden descendente de importancia. Mientras más relevante sea la arista y su dirección para el ajuste de la red, más grande es el índice de fuerza y el grosor de la flecha. 
Tabla 1

Tabla 1

Coeficientes de fuerza de las fechas de la red bayesiana por orden descendiente de importancia

\begin{tabular}{lll}
\hline \multicolumn{1}{c}{ Desde } & & Hacia \\
\hline Efic_Col & Autoefi & -71.85 \\
Participacion & Iden_Fem & -64.04 \\
Injusticia & Indignacion & -63.84 \\
Efic_Col & Expec & -45.28 \\
Autoefi & Norma_Sub & -41.55 \\
Norma_Sub & Participacion & -27.27 \\
Autoefi & Expec & -22.62 \\
Autoefi & Participacion & -14.71 \\
Injusticia & Efic_Col & -12.26 \\
Participacion & Indignacion & -8.75 \\
Efic_Col & Indignacion & -2.49 \\
Iden_Fem & Iden_Gen & -0.61 \\
Norma_Sub & Iden_Gen & -0.10 \\
Expec & Iden_Fem & -0.07
\end{tabular}

Nota. En esta tabla se puede observar de forma descendente el grado de fuerza de las aristas entre cada par de variables, mientras más grande el número de fuerza (independientemente si es negativo), mayor es la importancia de la arista para el adecuado ajuste de la red bayesiana.

\footnotetext{
Coeficientes de fuerza de las fechas de la red bayesiana por orden descendiente de importancia Nota. En esta tabla se puede observar de forma descendente el grado de fuerza de las aristas entre cada par de variables, mientras más grande el número de fuerza (independientemente si es negativo), mayor es la importancia de la arista para el adecuado ajuste de la red Bayesiana.
}

\section{Discusión}

Como se esperaba, en la red de correlaciones parciales, la autoeficacia, la eficacia colectiva y las expectativas de resultados se correlacionaron positivamente entre ellas. Al mismo tiempo la correlación más fuerte entre estas tres variables y la participación fue por parte de la autoeficacia, siendo congruente con la red bayesiana donde la eficacia colectiva influyó de manera indirecta en la participación por medio de la autoeficacia. Es decir, que por lo menos en los datos recabados, hay evidencia para suponer que la eficacia colectiva y las expectativas pudieran tener efecto en la participación en acciones colectivas por medio de la autoeficacia, 
esto es congruente con los resultados encontrados por Van Zomeren et al. (2013). De esta forma, aunque una mujer perciba que determinada acción colectiva es realizable y puede tener el efecto deseado, puede no participar en la misma si percibe que no tiene la capacidad para ello.

En la red de correlaciones parciales, la relación entre la indignación con la participación y falta de relación entre la percepción de injusticia y la participación es congruente con los resultados de la red bayesiana, donde la percepción de injusticia influyó indirectamente en la participación por medio de tres variables (eficacia colectiva, autoeficacia y norma subjetiva). Con los resultados encontrados, se puede suponer que en esta muestra las variables asociadas a la eficacia y a las normas subjetiva fueron determinantes para la participación. Por su parte, la indignación pudo ser resultado de la participación más que ser una causa. Sin embargo, autores como Van Zomeren et al. (2008), han encontraron que son las emociones asociadas a las creencias de injusticia las que pueden influir por encima de la percepción de injusticia para que una persona decida participar en determinada acción colectiva.

Por otra parte y en cuanto a las identidades se refiere, para Cameron y Lalone (2001) la identidad de género puede incluir, además del componente referido al sexo, las creencias relacionadas a los roles de género, de tal modo que una mujer con fuerte identidad de género se puede autodefinir como tradicional, no tradicional o feminista. De lo anterior se puede explicar que en la red de correlaciones parciales, la identidad de género este débilmente relacionada con la identidad feminista, siendo congruente con la influencia débil de la identidad feminista y la norma subjetiva hacia la identidad de género de la red bayesiana. Este resultado pudiera sugerir que las mujeres de la muestra pudieron tomar conciencia de género y de sus implicaciones derivado de la exposición al feminismo (Hasan \& Gill, 2016) y por cierta presión social por parte de amigas o familiares.

Específicamente en la red Bayesiana, es importante destacar la relación indirecta entre la injusticia y la participación, esta relación puede dar evidencias de que la injusticia percibida no basta por si misma para motivar a participar, es necesario que la persona considere que el grupo de mujeres puede realizar acciones colectivas (Klandermans, 1984), y presentar creencias de que se es capaz participar. Al mismo tiempo, la norma subjetiva puede ser un motivador extra que aunado a las anteriores variables para participar en acciones colectivas en el contexto de violencia hacia las mismas. Rees y Bamberg (2014) en su estudio en Alemania y en el contexto de la acción colectiva ante el cambio climático, encontraron que la norma subjetiva era un fuerte predictora de la intención a participar solamente en aquellas personas con alta identidad colectiva.

Contrario a lo que se hipotetizó, la participación no pareció ser influida por la identidad feminista ni por la identidad de género, sino por la injusticia de forma indirecta, esta relación se puede explicar dado que en México en 2019 y durante principios del 2020 el tema de la violencia hacia las mujeres fue tomando más relevancia en la medios tradicionales y en redes sociales (Lovera, 2020), lo que pudo crear un marco de 
injusticia generalizado en las mujeres, aún cuando no necesariamente presentaran identificación feminista o de género. $\mathrm{Al}$ mismo tiempo, las acciones colectivas durante estos años pudieron acercar a las mujeres a ideologías feministas y de género, lo que posiblemente aumentaría las probabilidades de presentar o aumentar dicha identidad después de haber participado en una marcha, protesta, en un colectivo u organización de mujeres. Al respecto, Radke et al. (2016), consideran que las mujeres pueden enfrentar una estigmatización al presentarse ante la sociedad como feministas, lo que pudiera provocar que explícitamente no expresen identificarse como feministas aún cuando están de acuerdo con esta ideología. Esto también podría explicar por qué en la red bayesiana la identidad feminista no influyó en la acción colectiva en contraste con otros resultados donde la identidad feminista ayudaba a predecir la participación en acciones colectivas (Liss et al., 2004; Nelson et al., 2008; Ramírez, 2018; Zucker, 2004).

En cuanto a la relación entre la percepción de injusticia y la eficacia colectiva, con base en los resultados de la red bayesiana, la percepción de injusticia aumentó las probabilidades de presentar eficacia colectiva y ésta influyó indirectamente sobre la participación. De acuerdo con Mackie et al. (2000), fuertes creencias de eficacia permitirían a las personas experimentar injusticia dado a que se tendrían más probabilidades de cambiar una situación en particular. Sin embargo, es probable que la exposición a un marco de injusticia difundido desde los colectivos feministas también incluya marcos de pronóstico o motivación (Simon \& Klandermans, 2001), lo que incluiría emociones y pensamientos asociados a la eficacia. Por su parte, Kelly y Breinlinger (1995) proponen que no es necesario una eficacia colectiva alta para que las mujeres participen colectivamente, pues la identidad social en sí misma pudiera ser suficiente para que éstas estén motivadas a participar y expresar valores personales. En este sentido, algunos autores (Sabucedo et al., 2018) han propuesto el constructo de obligación moral para explicar cómo las personas deciden participar en acciones colectivas aun cuando consideran que determinada acción no tendrá ningún tipo de éxito, pues la motivación a comportarse con base en las propias expectativas morales puede ser suficiente. Incluir la obligación moral en futuras investigaciones puede ser fundamental para entender la importancia de los valores y su expresión en la acción colectiva.

\section{Limitaciones del estudio}

En el presente estudio algunas escalas (las correspondientes a la autoeficacia y expectativas de resultados) mostraron índices de consistencia interna bajos, lo que puede comprometer los resultados. Es necesario seguir trabajando para contar con instrumentos válidos y confiables para medir las principales variables estudiadas en el contexto de la acción colectiva en contra de la violencia hacia las mujeres.

La obtención de datos por redes sociales pude implicar un problema para realizar generalizaciones de resultados y modelos. Tufekci (2014) 
considera que existen sesgos muestrales pues los usuarios de cada red social pueden ser cualitativamente diferentes. Además, es importante contar con muestras más grandes y representativas pues esto puede ayudar a generalizar este tipo de resultados. Por último, contrastar datos obtenidos por redes sociales y los obtenidos por medios tradicionales puede ayudar a conocer los posibles efectos de la obtención de los datos en por estos medios.

\section{Conclusión}

La percepción de injusticia (con su componente emocional) y las creencias relacionadas con la eficacia (autoeficacia, eficacia colectiva y expectativas de resultados) son unas de las variables a las que más se les ha prestado atención en el estudio de la acción colectiva, estudios como el presente ratifican su importancia en el contexto de violencia hacia las mujeres. Variables recientemente estudiadas como la obligación moral (Sabucedo et al., 2018) pudieran ayudar a explicar por qué algunas personas deciden participar en acciones colectivas aun cuando pueden considerarlas poco eficaces, prestar pocas emociones (positivas y negativas) y poca o nula norma subjetiva para involucrarse. De mismo modo, puede ser relevante separar la acción colectiva realizada offline de la online, pues los factores que motivan estos dos tipos de acciones pueden ser significativamente diferentes (Rodríguez \& González-García, 2018). Es conveniente considerar en este tipo de estudios emociones positivas asociadas a las creencias de eficacia como la esperanza (Wlodarczyk et al., 2017) o el empoderamiento (Drury \& Reicher, 2009) para indagar cuál es su papel para la explicación de la acción colectiva (Sabucedo \& Vilas, 2014) en este contexto.

Estudios como el presente evidencian la importancia de considerar múltiples variables cognitivas, emocionales, normativas, morales, etcétera, en el estudio de la acción colectiva para comprender cada vez mejor los diversos procesos y vías por las que las personas deciden participar y buscar resolver una problemática de forma colectiva. Utilizar algoritmos basados en datos puede ser de ayuda para una mejor compresión de estas variables y cómo se relacionan en contextos específicos. Es viable suponer que las relaciones entre las variables dependen fuertemente del contexto social. Por ejemplo, la identidad puede ser la variable que denote la participación o la participación derivada de una percepción de injusticia pudiera crear una identidad, al mismo tiempo, el tipo de emociones (positivas o negativas) pueden tener efectos diferenciadores en la acción colectiva dependiendo de la problemática (Sabucedo et al., 2017).

\section{Agradecimientos}

Se agradece al Consejo Nacional de Ciencia y Tecnología (CONACYT) por la beca que permitió el financiamiento de esta investigación, con número de becario 925505. Se declara que ninguno de los autores presenta un interés comercial o asociativo que presente un conflicto de 
intereses con el presente trabajo. Los datos de la presente investigación fueron usados para la presentación titulada "Miedo ante la violencia hacia las mujeres; resultados preliminares hacia un modelo de participación ciudadana” en el XIII Congreso de Posgrado en Psicología organizado por la UNAM.

\section{Referencias}

Ajzen, I. (1991). The theory of planned behavior. Organizational Behavior and Human Decision Processes, 50(2), 179-211. https://doi.org/10.1016/074 9-5978(91)90020-T

Bamberg, S., Rees, J., \& Seebauer, S. (2015). Collective climate action: Determinants of participation intention in community-based proenvironmental initiatives. Journal of Environmental Psychology, 43, 155-165. https://doi.org/10.1016/j.jenvp.2015.06.006

Beard, C., Millner, A. J., Forgeard, M. J., Fried, E. I., Hsu, K. J., Treadway, M. T., ... \& Björgvinsson, T. (2016). Network analysis of depression and anxiety symptom relationships in a psychiatric sample. Psychological Medicine, 46(16), 3359-3369. https://doi.org/10.1017/S003329171600 2300

Borsboom, D., \& Cramer, A. O. (2013). Network analysis: An integrative approach to the structure of psychopathology. Annual Review of Clinical Psychology, 9, 91-121. https://doi.org/10.1146/annurev-clinpsy-050212185608

Breinlinger, S., \& Kelly, C. (1994). Women's responses to status inequality: A test of social identity theory. Psychology of Women Quarterly, 18(1), 1-16. https://doi.org/10.1111/j.1471-6402.1994.tb00293.x

Cameron, J. E., \& Lalonde, R. N. (2001). Social identification and gender related ideology in women and men. British Journal of Social Psychology, 40(1), 59-77. https://doi.org/10.1348/014466601164696

Cerva Cerna, D. (2020). Activismo feminista en las universidades mexicanas: la impronta política de las colectivas de estudiantes ante la violencia contra las mujeres. Revista de La Educación Superior, 49(194), 137-157.

Chen, Y. Z. (2014). How to become a feminist activist after the institutionalization of the women's movements: The generational development of feminist identity and politics in Mexico City. Frontiers: $A$ Journal of Women Studies, 35(3), 183-206. https://doi.org/10.5250/fron jwomestud.35.3.0183

Clay-Warner, J. (2001). Perceiving procedural injustice: The effects of group membership and status. Social Psychology Quarterly, 224-238. https://do i.org/10.2307/3090113

Delgado, S. R., \& Arias, H. J. C. (2018). La acción colectiva de los jóvenes y la construcción de ciudadanía. Revista Argentina de Sociología, 6(11), 272-296.

Drury, J., \& Reicher, S. (2009). Collective psychological empowerment as a model of social change: Researching crowds and power. Journal of Social Issues, 65(4), 707-725. https://doi.org/10.1111/j.1540-4560.2009.0162 2.x 
Dunckel Graglia, A. (2013). 'Pink transportation' in Mexico City: Reclaiming urban space through collective action against gender-based violence. Gender \& Development, 21(2), 265-276. https://doi.org/10.1080/13552 074.2013 .802131

Dunckel Graglia, A. (2016). Finding mobility: Women negotiating fear and violence in Mexico City's public transit system. Gender, Place \& Culture, 23(5), 624-640. https://doi.org/10.1080/0966369X.2015.1034240

Eisele, H., \& Stake, J. (2008). The differential relationship of feminist attitudes and feminist identity to self-efficacy. Psychology of Women Quarterly, 32(3), 233-244. https://doi.org/10.1111/j.1471-6402.2008.00432.x

Epskamp, S., \& Fried, E. I. (2018). A tutorial on regularized partial correlation networks. Psychological Methods, 23(4), 617-634. https://doi.org/10.10 37/met0000167

Epskamp, S., Cramer, A. O. J., Waldorp, L. J., Schmittmann, V. D., \& Borsboom, D. (2012). Qgraph: Network visualizations of relationships in psychometric data. Journal of Statistical Software, 48(4), 1-18. https://do i.org/10.18637/jss.v048.i04

Folger, R. (1986). A referent cognitions theory of relative deprivation. En J. M. Olson, C. P. Herman \& M. P.Zanna (Eds.), Relative deprivation and social comparison: The Ontario symposium (pp. 217-242). Erlbaum.

Friedman, J., Hastie, T., \& Tibshirani, R. (2014). Glasso: Graphical lassoestimation of Gaussian graphical models. R Package Version, 1(8).

Gobierno de la Ciudad de México. (2019, noviembre). Se publica en gaceta oficial declaratoria de alerta por violencia de género. https://jefaturadegobierno.cdmx.gob.mx/comunicacion/nota/sepublica-en-gaceta-oficial-declaratoria-de-alerta-por-violencia-de-genero

Groenendyk, E. W., \& Banks, J. A. (2014). Emotional rescue: How affect helps partisans overcome collective action problems. Political Psychology, 35(3), 359-378. https://doi.org/10.1111/pops.12045

Hasan, V. F., \& Gil, A. S. (2016). La comunicación con enfoque de género, herramienta teórica y acción política. Medios, agenda feminista y prácticas comunicacionales. El caso de Argentina. La Ventana. Revista de Estudios de Género, 5(43), 246-280.

Hincapié, S. (2017). Acción colectiva de las mujeres y derechos humanos en México. Movilizando el dolor en medio del conflicto armado. Revista Estudios Socio-Jurídicos, 19(2), 97-127.

Kelly, C., \& Breinlinger, S. (1995). Identity and injustice: Exploring women's participation in collective action. Journal of Community \& Applied Social Psychology, 5(1), 41-57. https://doi.org/10.1002/casp.2450050104

Kelly, C., \& Breinlinger, S. (1996). The social psychology of collective action: Identity, injustice and gender. Taylor \& Francis US.

Klandermans, B. (1984). Mobilization and participation: Social-psychological expansions of resource mobilization theory. American Sociological Review, 49(5), 583-600. https://doi.org/10.2307/2095417

Laboratorio de Análisis de Organizaciones y Movimientos Sociales (CEIICHUNAM). (2020). Protesta social en México, mujeres en las calles. http://1 aoms.org/ 
Lagarde, M. (2011). Prefacio: Claves feministas en torno al feminicidio: construcción teórica, política y jurídica. En R. -L. Fregoso \& C. Bejarano (Eds.), Feminicidio en Ame\#rica Latina. UNAM.

Leach, C. W., Iyer, A., \& Pedersen, A. (2006). Anger and guilt about ingroup advantage explain the willingness for political action. Personality and Social Psychology Bulletin, 32(9), 1232-1245. https://doi.org/10.1177/01 46167206289729

Liss, M., Crawford, M., \& Popp, D. (2004). Predictors and correlates of collective action. Sex Roles, 50, 771-779. https://doi.org/10.1023/B:SER S.0000029096.90835.3f

Lovera, S. (2020, septiembre). México: Balance sobre la violencia contra las mujeres. AmecoPress. https://amecopress.net/Mexico-Balance-violenciacontra-las-mujeres

Mackie, D. M., Devos, T., \& Smith, E. R. (2000). Intergroup emotions: Explaining offensive action tendencies in an intergroup context. Journal of Personality and Social Psychology, 79(4), 602-616. https://doi.org/10.1 037/0022-3514.79.4.602

Mackie, D. M., Smith, E. R., \& Ray, D. G. (2008). Intergroup emotions and intergroup relations. Social and Personality Psychology Compass, 2(5), 1866-1880. https://doi.org/10.1111/j.1751-9004.2008.00130.x

Madrid, Y. C. G. (2019). La alerta de violencia de género en el Estado de México. Una reflexión sobre su implementación. UVserva, (8), 143-157. https://d oi.org/10.25009/uvserva.v0i8.2642

Mair, P. (2018). Modern psychometrics with $R$. Springer International Publishing. https://doi.org/10.1007/978-3-319-93177-7

McNally, R. J., Mair, P., Mugno, B. L., \& Riemann, B. C. (2017). Co-morbid obsessive-compulsive disorder and depression: A Bayesian network approach. Psychological Medicine, 47(7), 1204-1214. https://doi.org/10.1 017/S0033291716003287

Nelson, J. A., Liss, M., Erchull, M. J., Hurt, M. M., Ramsey, L. R., Turner, D. L., \& Haines, M. E. (2008). Identity in action: Predictors of feminist selfidentification and collective action. Sex Roles, 58(9), 721-728. https://do i.org/10.1007/s11199-007-9384-0

Pedraza, Y. R. (2019). La violencia contra la mujer: una emergencia nacional. Letras Jurídicas: Revista Electrónica de Derecho, (28), 1870-2155.

Polletta, F., \& Jasper, J. M. (2001). Collective identity and social movements. Annual Review of Sociology, 27(1), 283-305. https://doi.org/10.1146/an nurev.soc.27.1.283

Poma, A., \& Gravante, T. (2013). Las emociones como factor explicativo en las experiencias de protesta y resistencia social. Una propuesta de categorización y organización. III Coloquio de Investigación. Las emociones en el marco de las ciencias sociales: perspectivas interdisciplinarias.

Radke, H. R. M., Hornsey, M. J., \& Barlow, F. K. (2016). Barriers to women engaging in collective action to overcome sexism. American Psychologist, 71(9), 863-874. https://doi.org/10.1037/a0040345

Ramírez, L. C. C. (2018). La acción colectiva feminista, ¿ de la lucha de clases a la lucha de géneros? Apuntes para la comprensión/práctica de los movimientos sociales, en torno al caso "ni una menos". Ciencia Política, 13(26), 19-61. 
Rees, J. H., \& Bamberg, S. (2014). Climate protection needs societal change: Determinants of intention to participate in collective climate action. European Journal of Social Psychology, 44(5), 466-473. https://doi.org/10 $.1002 /$ ejsp.2032

Rodríguez, A. P., \& González-García, R. (2018). La incidencia de la acción colectiva feminista virtual en las respuestas del estado a la violencia de género en México. Edäbi Boletín Cientifico de Ciencias Sociales y Humanidades del ICSHu, 7(13), 28-37. https://doi.org/10.29057/icshu .v7i13.3490

Romero Mendoza, M. P., Gómez-Dantes, H., Manríquez Montiel, Q., Saldívar Hernández, G. J., Campuzano Rincón, J. C., Lozano, R., \& Medina-Mora Icaza, M. E. (2021). The invisible burden of violence against girls and young women in Mexico: 1990 to 2015. Journal of Interpersonal Violence, 36(5-6), 2753-2771. https://doi.org/10.1177/0886260517753851

Sabucedo, J. M., \& Vilas, X. (2014). Anger and positive emotions in political protest. Universitas Psychologica, 13(3), 829-838. http://dx.doi.org/10.11 144/Javeriana.UPSY13-3.apep.

Sabucedo, J. M., Dono, M., Alzate, M. y Seoane, G. (2018). The importance of protesters' moral: Moral obligation as a key variable to understand collective action. Frontiers in Psychology, 9, 1-12. https://doi.org/10.338 9/fpsyg.2018.00418

Sabucedo, J. M., Durán, M., \& Alzate, M. (2010). Identidad colectiva movilizada. Revista de Psicología Social, 25(2), 189-201. https://doi.org/1 $0.1174 / 021347410791063822$

Sabucedo, J. M., Gómez-Román, C., Alzate, M., Van Stekelenburg, J., \& Klandermans, B. (2017). Comparing protests and demonstrators in times of austerity: Regular and occasional protesters in universalistic and particularistic mobilisations. Social Movement Studies, 16(6), 704-720. ht tps://doi.org/10.1080/14742837.2017.1338940

Sancho, G. R. (2018). El devenir feminista de la acción colectiva: las redes digitales y la política de prefiguración de las multitudes conectadas. Teknokultura, 15(2), 223-240.

Scutari, M. (2010). Learning Bayesian networks with the BNLEARN package. Journal of Statistical Software, 35(3), 1-22. arXiv:0908.3817

Scutari, M., \& Denis, J. B. (2015). Bayesian networks with examples in R. CRC Press.

Simon, B., \& Klandermans, B. (2001). Politicized collective identity: A social psychological analysis. American Psychologist, 56(4), 319-331. https://do i.org/10.1037/0003-066X.56.4.319

Sosa, M. (2016, octubre). América Latina grita 'Ni una menos': las protestas contra los feminicidos toman las capitales. Univisión Noticias. http://www.univision.com/noticias/violaciones/america-latina grita-ni-una-menos-las-protestas-contra-los-feminicidios-toman-las-capi tales

Stümer, S., \& Simon, B. (2009). Pathways to collective protest: calculation, identification, or emotion? A critical analysis of the role of group-based anger in social movement participation. Journal of Social Issues, 65(4), 681-705. https://doi.org/10.1111/j.1540-4560.2009.01620.x

Stürmer, S., \& Simon, B. (2004). The role of collective identification in social movement participation: A panel study in the context of the German gay 
movement. Personality and Social Psychology Bulletin, 30(3), 263-277. ht tps://doi.org/10.1177/0146167203256690

Tajfel, H. (1978). Social categorization, social identity, and social comparison. En H. Tajfel (Ed.), Differentiation between social groups (pp. 61-76). Academic Press.

Thomas, E. F., Mavor, K. I., \& McGarty, C. (2012). Social identities facilitate and encapsulate action-relevant constructs: A test of the social identity model of collective action. Group Processes \& Intergroup Relations, 15(1), 75-88. https://doi.org/10.1177/1368430211413619

Tufekci, Z. (2014, mayo). Big questions for social media big data: Representativeness, validity and other methodological pitfalls. En Eighth international AAAI conference on weblogs and social media.

Van Stekelenburg, J., Klandermans, B., \& Van Dijk, W. W. (2009). Context matters: Explaining how and why mobilizing context influences motivational dynamics. Journal of Social Issues, 65(4), 815-838. https://d oi.org/10.1111/j.1540-4560.2009.01626.x

Van Zomeren, M., Postmes, T., \& Spears, R. (2008). Toward an integrative social identity model of collective action: A quantitative research synthesis of three socio-psychological perspectives. Psychological Bulletin, 134(4), 504-535. https://doi.org/10.1037/0033-2909.134.4.504

Van Zomeren, M., Saguy, T., \& Schellhaas, F. M. (2013). Believing in "making a difference" to collective efforts: Participative efficacy beliefs as a unique predictor of collective action. Group Processes \& Intergroup Relations, 16(5), 618-634. https://doi.org/10.1177/1368430212467476

Van Zomeren, M., Spears, R., Fischer, A. H., \& Leach, C. W. (2004). Put your money where your mouth is! Explaining collective action tendencies through group-based anger and group efficacy. Journal of Personality and Social Psychology, 87(5), 649 -664. https://doi.org/10.1037/0022-3514. 87.5.649

Wlodarczyk, A., Basabe, N., Páez, D., \& Zumeta, L. (2017). Hope and anger as mediators between collective action frames and participation in collective mobilization: The case of 15-M. Journal of Social and Political Psychology, 5(1), 200-223. http://dx.doi.org/10.23668/psycharchives.1759

Zucker, A. N. (2004). Disavowing social identities: What it means when women say, "I'm not a feminist, but...". Psychology of Women Quarterly, 28(4), 423-435. https://doi.org/10.1111/j.1471-6402.2004.00159.x 